\title{
On bases and the shift operator
}

by

J. R. HOLUB (Blacksburg, Va)

If $X$ is a symmetric Banach sequence space, $S$ the shift operator on $X$, and $x_{0}$ a finitely non-zero sequence, characterizations for basic sequences (and bases) in $X$ of the form $\left\{S^{n} x_{0}\right\}_{n=0}^{\infty}$ are given in terms of the behavior of zeros of the polynomial $p(z)$ associated with $x_{0}$. A fundamental difference is shown to separate the case $X=l^{1}$ from all others and this difference is discussed in detail. Additional results including characterization of subspaces of the form $\left[S^{n} x_{0}\right]$ in $X$ and remarks on the more general situation in which $x_{0}$ is an arbitrary member of $\eta^{1}$ are also given.

\$1. Introduction. Let $\mathscr{F}$ denote the set of all finitely non-zero complex sequences and $\left\{e_{n}\right\}_{n=0}^{\infty}$ the sequence in $\mathscr{F}$ defined by $e_{0}=(1,0,0, \ldots), e_{1}$ $=(0,1,0, \ldots)$, etc. If $\Phi$ is any symmetric norming function defined on $\mathscr{F}$ ([1], p. 71], then the set $X$ of all complex sequences $\left\{a_{n}\right\}_{n=0}^{\infty}$ for which $\lim \sup \Phi\left\{a_{n}\right\}_{n=N+1}^{N+M}=0$ is a Banach space having $\left\{e_{n}\right\}_{n=0}^{\infty}$ as a symmetric $\lim _{N \rightarrow \infty} \operatorname{sum}_{M \rightarrow}$

basis. Particular examples include the well known $l^{p}$-spaces $(1 \leqslant p<+\infty)$, $c_{0}$, Orlicz spaces [4], Lorentz spaces [4], the spaces $G_{I I}$ and $G_{\pi}$ of MacaevGohberg-Krein [1], etc. For each such space $X$ the shift operator $S$ defined by $S e_{n}=e_{n+1}, n=0,1,2, \ldots$, is an isometry of co-rank 1 whose spectrum is the closed unit disc in the complex plane.

A very natural and interesting problem which suggests itself is the following:

If $x_{0}=\left(1, a_{1}, a_{2}, \ldots\right)$ is in $X$, when is $\left\{S^{n} x_{0}\right\}_{n=0}^{\infty}$ a basio sequence (or even a basis) in $X$ ?

For an arbitrary sequence $x_{0}$ in $X$ the solution of this problem is known only in the case $X=l^{2}$ [2]. In this paper we solve the problem for the special case where $x_{0}$ is finitely non-zero. The most interesting and significant aspect of the solution is the way in which it distinguishes the space $l^{1}$ from all other spaces $X$.

In particular, if $X \neq l^{1}$ and $x_{0}=\left(1, a_{1}, a_{2}, \ldots, a_{N}, 0,0, \ldots\right)$, wo show tho following are equivalent:

(i) $\left\{S^{n} x_{0}\right\}_{n=0}^{\infty}$ is a basic sequence in $X$.

(ii) $\left\{S^{n} x_{0}\right\}_{n=0}^{\infty}$ is a basic sequence in $X$ which is similar to $\left\{\theta_{n}\right\}_{n=0}^{\infty}$.

(iii) The polynomial $p(z)=1+a_{1} z+\ldots+a_{N} z^{N}$ has no zero on the circle $|z|=1$. 
That is, the basic sequence properties of $\left\{S^{n} x_{0}\right\}_{n=0}^{\infty}$ are completely determined by the existence or non-existence of zeros of $p(z)$ on the circle $|z|=1$ and every basic sequence $\left\{S^{n} x_{0}\right\}_{n=0}^{\infty}$ is (isomorphically) the same as $\left\{e_{n}\right\}_{n=0}^{\infty}$. The situation in $l^{1}$ is considerably more complicated, as the example of the basic sequence $\left\{S^{n} x_{0}\right\}_{n=0}^{\infty}$ in $l^{1}$ with $x_{0}=(1,-1,0,0, \ldots)$ indicates. Yet even here we can characterize the basicity of $\left\{S^{n} x_{0}\right\}_{n=0}^{\infty}$ in terms of $p(z)$. Namely, we show that $\left\{S^{n} x_{0}\right\}_{n=0}^{\infty}$ is basic in $l^{1}$ if and only if $p(z)$ has no zero of multiplicity greater than one on the circle $|z|=1$. and that, in this case, the isomorphic type of the basic sequence $\left\{S^{n} x_{0}\right\}$ is completely determined by the zeros of $p(z)$ on $|z|=1$. Thus thoro is a richness in the theory for $X=l^{1}$ that is missing in the other ases and which we explore in some detail. Related problems and extensions of these results are also discussed.

§2. Preliminaries. If $\left\{x_{n}\right\}_{n=0}^{\infty}$ is a sequence in a Banach space $X$, the closed linear span of $\left\{x_{n}\right\}$ is denoted by $\left[x_{n}\right]$. The sequence $\left\{x_{n}\right\}$ is said to be minimal if $x_{n} \notin\left[x_{i}\right]_{i \neq n}$ for all $n$. It is easy to see that $\left\{x_{n}\right\}$ is minimal if and only if there is a sequence $\left\{f_{n}\right\}_{n=0}^{\infty} \subset X^{*}$ for which $\left\langle f_{n}, x_{m}\right\rangle=\delta_{n m}$ for all $m, n$. In this case $\left\{f_{n}\right\}$ is called a biorthogonal sequence to $\left\{x_{n}\right\}$ and tho system $\left\{x_{n}, f_{n}\right\}$ a biorthogonal system. For simplicity, we often omit explicit mention of $\left\{f_{n}\right\}$ and say " $\left\{x_{n}\right\}$ is a biorthogonal system". If sup $\left\|x_{n}\right\|<+\infty$ and $\sup _{n}\left\|f_{n}\right\|<+\infty$, we call $\left\{x_{n}, f_{n}\right\}$ a bounded biorthogonal system. A sequence $\left\{x_{n}\right\}$ in $X$ is called a basic sequence if it forms a Schauder basis for $\left[x_{n}\right]$. Two basic sequences $\left\{x_{n}\right\}$ and $\left\{y_{n}\right\}$ are said to be similar if there is an invertible operator $T:\left[x_{n}\right] \rightarrow\left[y_{n}\right]$ for which $T x_{n}=y_{n}$

It is well known that if $\left\{x_{n}\right\}$ is a basic sequence in $X$ for which $0<\inf \left\|x_{n}\right\| \leqslant \sup \left\|x_{n}\right\|<+\infty$, there is a biorthogonal sequence $\left\{f_{n}\right\}$ in $X^{*}$ for which $\sup _{n}\left\|f_{n}\right\|<+\infty$, i.e. $\left\{x_{n}\right\}$ is a bounded biorthogonal system in $X$. In certain cases the converse is also true. The following simple generalization of a result of Szankowski and Zippin [6] is crucial to all of our work and gives a sufficient criterion for this phenomona to oceur.

THEOREM 2.1 [6]. Let $\left\{e_{n}\right\}_{n=0}^{\infty}$ be a basis for a Banach space $X$ for which $0<\inf \left\|e_{n}\right\| \leqslant \sup \left\|e_{n}\right\|<+\infty$, let 7 be a positive integer, and for oach $n=n^{n}, 1,2, \ldots$ let $x_{n}=e_{n}+a_{1}^{(n)} e_{n+1}+\ldots+a_{k}^{(n)} e_{n+k}$. Then $\left\{x_{n}\right\}_{n \rightarrow \infty}^{\infty}$ is a basic sequence in $X$ if and only if it is a bounded biorthogonal system.

Two other results which are well known, and which wo will tuso repeatedly, are:

LEMMA 2.2. If $\left\{x_{n}\right\}_{n=0}^{\infty}$ is a sequence in a Banaoh spaco $X, T$ a bounded linear operator on $X$, and $\left\{T x_{n}\right\}_{n=0}^{\infty}$ a bounded biorthogonal system in $X$, then $\left\{x_{n}\right\}$ is a bounded biorthogonal system in $X$.
LEMMA 2.3. Let $X$ be a separable symmetric Banach sequence space and $S$ the shift operator on $X$. Then

(i) If $|\lambda|>1$, the operator $\lambda I-S$ is invertible.

(ii) If $|\lambda|<1$, the operator $\lambda I-S$ is bounded below on $X$ but codim ran $(\lambda I-S)=1$.

(iii) $X_{f}|\lambda|=1$, the operator $\lambda I-S$ is not bounded below on $X$.

If $X \neq l^{1}, \operatorname{ran}(\lambda I-S)$ is dense in $X$.

If $X=l^{1}, \operatorname{codim} \operatorname{ran}(\lambda I-S)=1$.

For simplieity we adopt some uniform symbolism to be used throughout the paper. The symbol $X$ will always denote a separable symmetric Banach sequence space, $\left\{e_{n}\right\}$ the ustal anit vector basis for $X, x_{0}$ the finitely non-zero sequence $\left(1, a_{1}, a_{2}, \ldots, a_{N}, 0,0, \ldots\right)$, and $p(z)$ the $N$ th degree polynomial $1+a_{1} z+\ldots+a_{N} z^{N}$.

\$ 3. Main results. Given the space $X$ and a finitely non-zero sequence $x_{0}$, we want to characterize when $\left\{S^{n} x_{0}\right\}_{n=0}^{\infty}$ is a basic sequence in $X$. We begin by settling the simplest of such questions, that of the similarity to the basis $\left\{e_{n}\right\}_{n=20}^{\infty}$ for $X$.

THEonem 3.1. If $x_{0}=\left(1, a_{1}, \ldots, a_{N}, 0,0, \ldots\right)$, the sequence $\left\{S^{n} x_{0}\right\}_{n=0}^{\infty}$ is a basie sequence in $X$ which is similar to $\left\{e_{n}\right\}_{n=0}^{\infty}$ if and only if $p(z)=1+$ $+a_{1} z+\ldots+a_{N} z^{N}$ has no zero on the circle $|z|=1$.

Proof. Let $T=I+a_{1} S+a_{2} S^{2}+\ldots+a_{N} S^{N}$. Then $T$ is a bounded linear operator on $X$ and $T e_{n}=S^{n} x_{0}$ for all $n=0,1,2, \ldots$ If $p(z)$ factors as $p(z)=\left(1-b_{1} z\right)\left(1-b_{2} z\right) \ldots\left(1-b_{N} z\right)$, then certainly $T$ has the factorization $T=\left(I-b_{1} S\right)\left(I-b_{2} S\right) \ldots\left(I-b_{N} S\right)$.

Suppose $p(z)$ has no zero on the circle $|z|=1$. Then $\left|b_{i}\right| \neq 1$ for all $i$ and so, by Lemma 2.3, each factor $I-b_{i} S$ of $T$ is bounded below on $X$. It follows that $T: X \rightarrow\left[S^{n} x_{0}\right]_{n=0}^{\infty}$ is invertible and since $T e_{n}=S^{n} x_{0}$ for all $n$, we see that $\left\{S^{n} x_{0}\right\}_{n=0}^{\infty}$ is a basic sequence in $X$ which is similar to $\left\{e_{n}\right\}_{n=0}^{\infty}$.

Conversely, if $p(\approx)$ has a zero on the circle $|z|=1$, then in the above factorization of $T$ at least one $b_{i}$ has modulus 1 . By Lemma 2.3, the corresponding factor $I-b_{i} S$ is not bounded below on $X$, and since the factors of $T$ commute, it follows that $T$ itself is not bounded below on $X$. Therefore since $T o_{n}=S^{n} x_{0}$, wo see that $\left\{S^{n} x_{0}\right\}_{n=0}^{\infty}$ cannot be similar to $\left\{e_{n}\right\}_{n=0}^{\infty}$ and the theorem is proved.

Theorem 3.1 sliows that in studying the sequence $\left\{S^{n} x_{0}\right\}_{n=0}^{\infty}$, the only situation having real interest is that in which the polynomial $p(z)$ has at loasti one zero on the unit circle $|z|=1$. It is here that a fundamental difference occurs between the space $l^{1}$ and all other spaces $X$. Though Theorem 3.1 does not rule out the possibility that $\left\{S^{n} x_{0}\right\}_{n=0}^{\infty}$ may be a basic sequence oven though $p(z)$ has a zero on the unit circle, in fact this cannot occur except in the space $l^{1}$. 
THeorem 3.2. Suppose $X \neq l^{1}$ and $x_{0}=\left(1, a_{1}, \ldots, a_{N}, 0,0, \ldots\right)$ Then the following are equivalent:

(i) $\left\{S^{n} x_{0}\right\}$ is a bounded biorthogonal system in $X$.

(ii) $\left\{S^{n} x_{0}\right\}$ is a basic sequence in $X$.

(iii) $\left\{S^{n} x_{0}\right\}$ is a basic sequence in $X$ whioh is similar to $\left\{e_{n}\right\}$.

(iv) The polynomial $p(z)=1+a_{1} z+\ldots+a_{N} z^{N}$ has no zero on the circle $|z|=1$.

Proof. Clearly (iii) $\Rightarrow$ (ii) $\Rightarrow$ (i), and (iv) $\Rightarrow$ (iii) by Theorem 3.1. Thus we need only to show that (i) $\Rightarrow$ (iv).

Suppose $\left\{S^{n} x_{0}\right\}$ is a bounded biorthogonal system in $X$ but $p(z)$ has a zero on the circle $|x|=1$. Then, as in the proof of Theorom 3.1, the operator $T=I+a_{1} S+\ldots+a_{N} S^{N}$ has the factorization $T=\left(I-b_{1} S\right)$ $\left(I-b_{2} S\right) \ldots\left(I-b_{N} S\right)$, where we may assume $\left|b_{N}\right|=1$. Since $T e_{n}=S^{n} x_{0}$ for all $n$ and by assumption $\left\{S^{n} x_{0}\right\}$ is a bounded biorthogonal system, Lemma 2.2 implies that the sequence $\left\{\left(I-b_{N}\right) e_{n}\right\}$ is also a bounded biorthogonal system in $X$.

Now since $\left|b_{N}\right|=1$ and we are assuming $X \neq 1$, by Lemma 2.3 we have that the range of $I-b_{N} S$ is dense in $X$. One easily checks that the sequence $\left\{g_{n}\right\}_{n=0}^{\infty} \subset X^{*}$ defined by

$$
g_{n}=\left(b_{N}^{n}, b_{N}^{n-1}, \ldots, b_{N}, 1,0,0, \ldots\right)
$$

is biorthogonal to $\left\{\left(I-b_{N} S\right) e_{n}\right\}_{n=0}^{\infty}$ and since $\left[\left(I-b_{N} S\right) e_{n}\right]=X$, it must be the only such biorthogonal sequence. Therefore we have $\sup _{n}\left\|g_{n}\right\|_{X^{*}}$ $<+\infty$. Since $\left|b_{N}\right|=1$ and the norm on $X^{*}$ is symmetric, there is a positive number $K$ for which $\left\|\left(\varepsilon_{0}, \varepsilon_{1}, \ldots, \varepsilon_{m}, 0,0, \ldots\right)\right\|_{X^{*}} \leqslant K$ for all $\left|\varepsilon_{i}\right|=1$ and all $m=0,1,2, \ldots$ [1], p. 71. It follows that for any $\sum_{i=1}^{n} c_{i} e_{i}$ in $X$, $\left\|\sum_{i=1}^{n} e_{i} e_{i}\right\|_{X^{*}} \geqslant \frac{1}{K} \sum_{i=1}^{n}\left|e_{i}\right|$. That is, the operator $U: X \rightarrow l^{1}$ defined by $U e_{i}=e_{i}$ is continuous, implying $X$ is a subset of $l^{1}$ and consequently that $X=l^{1}$. But this contradicts our original assumption, and the proof is complete.

Remark. Theorem 3.2 should be compared with results of Szankowski and Zippin [6] which are of a similar nature.

As Theorem 3.2 shows, if $X \neq l^{1}$, the basic sequences in $X$ of the form $\left\{S^{n} x_{0}\right\}$ are all essentially the same, being similar to $\left\{e_{n}\right\}$. However, in $l^{1}$ the situation is much more interesting. For exmmple, if $x_{0}=(1,-1$, $0,0, \ldots)$, then it is well known and casy to check that $\left\{S^{n} x_{0}\right\}$ is a busio sequence in $l^{1}$ even though the associated polynomial $p(z)=1-\approx$ has a zero on the circle $|z|=1$. On the other hand, if $x_{0}=(1,-2,1,0,0, \ldots)$, then the polynomial $p(z)=1-2 z+z^{2}$ has only 1 as a zero but $\left\{S^{n} x_{0}\right\}$ is not a basic sequence. We will now show that the determining factor in whether $\left\{S^{n} x_{0}\right\}$ is basic in $l^{1}$ is the multiplicity of the zeros of $p(z)$ on the circle $|z|=1$.

Before we begin, it is necessary to analyze in more detail the general form of sequences in $X^{*}$ which are biorthogonal to the sequence $\left\{S^{n} x_{0}\right\}$ in $X$. If wo again set $x_{0}=\left(1, a_{1}, a_{2}, \ldots, a_{N}, 0,0, \ldots\right), p(z)=1+a_{1} z+$ $+a_{2} z^{2}+\ldots+a_{N} z^{N}$, and $p^{-1}(z)=1+o_{1} z+c_{2} z^{2}+\ldots$, then since $p(z)$. . $p^{-1}(z)=1$ (for suitable $z$ !), we must have that $a_{n}+a_{n-1} c_{1}+\ldots+a_{1} c_{n-1}+$ $+c_{n}=0$ for all $n \geqslant 1$. Oonsequently, the sequence $\left\{f_{n}\right\}_{n=0}^{\infty}$ in $X^{*}$ defined by $f_{n}=\left(o_{n}, o_{n-1}, \ldots, o_{1}, 1,0,0, \ldots\right)$ is biorthogonal to $\left\{S^{n} x_{0}\right\}_{n=0}^{\infty}$. We call $\left\{f_{n}\right\}$ the oanonioal biorthogonal sequence to $\left\{S^{n} x_{0}\right\}$ (since if $\left[S^{n} x_{0}\right]=X$, the sequence $\left\{f_{n}\right\}$ is the only such bioxthogonal sequence). If $\left\{g_{n}\right\}_{n=0}^{\infty}$ is any sequence in the annihilator subspace $\left[S^{n} x_{0}\right]^{\perp} \subset X^{*}$, then the sequence $\left\{f_{n}+g_{n}\right\}_{n=0}^{\infty}$ is again biorthogonal to $\left\{S^{n} x_{0}\right\}_{n=0}^{\infty}$ and, moreover, every sequence biorthogonal to $\left\{S^{n} w_{0}\right\}$ is of this form for some $\left\{g_{n}\right\} \subset\left[S^{n} x_{0}\right]^{\perp}$. Thus we note that $\left\{S^{n} w_{0}\right\}$ is a bounded biorthogonal system in $X$ if and only if there is a sequence $\left\{g_{n}\right\}_{n=0}^{\infty} \subset\left[S^{n} \infty_{0}\right]^{\perp-}$ for which $\sup \left\|f_{n}+g_{n}\right\|_{X^{*}}<+\infty$.

Romark. The construction given here for the general form of biorthogonal sequences to $\left\{S^{n} x_{0}\right\}$ is clearly also valid in the more general setting in which $x_{0}$ is an arbitrary member of $X$. This observation is often very useful in treating problems more general than those considered here (seo, 0.g., \$ 4 ).

To characterize the basic sequences $\left\{S^{n} x_{0}\right\}$ in $l^{1}$, it is helpful to first provo at special case.

LEMms 3.3. If $x_{0}=\left(1,-2 a, a^{2}, 0,0, \ldots\right)$ with $|a|=1$, then $\left\{S^{n} x_{0}\right\}_{n=0}^{\infty}$ is not a bounded biorthogonal system in $l^{1}$.

Proof. First, wo claim that $\left[S^{n} x_{0}\right]^{\perp}=\left[\left(1,1 / a, 1 / a^{2}, \ldots\right)\right] \subset l^{\infty}$. For clearly $\left(1,1 / a, 1 / a^{2}, \ldots\right)$ is a member of $l^{\infty}$ and is in $\left[S^{n} x_{0}\right]^{\perp}$ sinco $1 / a$ is a root of the polynomial $z^{n}\left(1-2 a z+a^{2} z^{2}\right)$ for all $n=0,1,2, \ldots$ If $\left[S^{n} x_{0}\right]$ wore of dimension $\geqslant 2$, then there would exist an element in $\left[S^{n} x_{0}\right]^{\perp}$ $\left[S^{n} x_{0}\right]$ wore of $\left(0,1, b_{1}, b_{2}, \ldots\right)$. For this to be so, one easily checks that $b_{n}=(n+1) / a^{n}$ for all $n=1,2, \ldots$ However, since $|a|=1$, it follows that $\left|b_{n}\right|=n+1$ for all $n$, a contradiction to the fact that $\left(0,1, b_{1}, b_{2}, \ldots\right)$ is in $l^{\infty}$. Honce it must bo that $\left[S^{n} x_{0}\right]^{\perp}$ is one-dimensional and is spanned by the sequenee $\left(1,1 / a, 1 / a^{2}, \ldots\right)$.

Now $p(z)=1-2 a z+a^{2} z^{2}$ so

$$
p^{-1}(z)=1+\frac{2}{a} z+\frac{3}{a^{2}} z^{2}+\cdots
$$

According to our preceding remarks, the canonical biorthogonal sequence $\left\{f_{n}\right\}$ associated with $\left\{S^{n} x_{0}\right\}$ is defined by

$$
f_{n}=\left((n+1) / a^{n}, n / a^{n-1}, \ldots, 2 / a, 1,0,0, \ldots\right)
$$

and any biorthogonal sequence $\left\{h_{n}\right\}$ to $\left\{S^{n} x_{0}\right\}$ is of the form $\left\{f_{n}+g_{n}\right\}$ 
for some sequence $\left\{g_{n}\right\} \subset\left[S^{n} x_{0}\right]^{\perp}$. That is, $h_{n}=f_{n}+\lambda_{n}\left(1,1 / a, 1 / a^{2}, \ldots\right)$ $=\left((h+1) / a^{n}+\lambda_{n}, \ldots, 1+\lambda^{n} / a^{n}, \lambda_{n} / a^{n+1} \ldots\right)$ for some sequence of scalars $\left\{\lambda_{n}\right\}$. Since $|a|=1$, it is obvious that for any such $\left\{h_{n}\right\}$ we have $\left\|h_{n}\right\|_{\infty}$ $\geqslant(n+1) / 2$ for all $n$ and therefore $\left\{S^{n} x_{0}\right\}$ cannot be a bounded biorthogonal sequence in $l^{1}$.

From this we get the following fundamental characterization of basic sequences $\left\{S^{n} x_{0}\right\}$ in $l^{1}$.

Theorem 3.4. If $x_{0}=\left(1, a_{1}, a_{2}, \ldots, a_{N}, 0,0, \ldots\right)$, then $\left\{S^{n} x_{0}\right\}_{n=0}^{\infty}$ is a basic sequence in $l^{1}$ if and only if the polynomial $p(z)=1+a_{1} z+\ldots+a_{N} z^{N}$ has no zero of multiplicity greater than 1 on the unit oircle $|z|=1$.

Proof. As in the proof of Theorem 3.1, let $T=I+a_{1} S+\ldots+a_{N} S^{N}$ and suppose $T$ factors as $T=\left(I-b_{1} S\right)\left(I-b_{2} S\right) \ldots\left(I-b_{N} S\right)$, where $\left\{1 / b_{i}\right\}_{i=1}^{N}$ is the set of the $N$ roots of $p(z)$ counted according to multiplicity.

If $p(z)$ has a zero of multiplicity $\geqslant 2$ on the circle $|z|=1$, then in the factorization of $T$ we may assume $b_{N-1}=b_{N}$ and $\left|b_{N-1}\right|=\left|b_{N}\right|=1$. By Lemma 3.3, the sequence $\left\{\left(I-b_{N-1} S\right)\left(I-b_{N} S\right) e_{n}\right\}=\left\{S^{n}\left(1,-2 b_{N}\right.\right.$, $\left.\left.b_{N}^{2}, 0,0, \ldots\right)\right\}$ is not a bounded biorthogonal system in $l^{1}$. Since $\left\{S^{n} x_{0}\right\}$ $=\left\{T e_{n}\right\}=\left\{\left[\left(I-b_{1} S\right) \ldots\left(I-b_{N-2} S\right)\right]\left(I-b_{N-1} S\right)\left(I-b_{N} S\right) e_{n}\right\}$, it follows from Lemma 2.2 that $\left\{S^{n} x_{0}\right\}$ is not a bounded biorthogonal system in $l^{1}$ and hence not a basic sequence.

On the other hand, suppose $p(z)$ has no zero of multiplicity greater than 1 on $|z|=1$. Those factors of $T$ of the form $I-b_{i} S$ with $\left|b_{i}\right| \neq 1$ aro all bounded below on $l^{1}$ and hence do not affect whether or not $\left\{S^{1 n} x_{0}\right\}$ is a basic sequence. For this reason we may assume the $N$ roots of $p(z)$ are all distinct and all lie on the circle $|z|=1$.

Under this assumption, let $\left\{z_{i}\right\}_{i=1}^{N}$ denote the roots of $p(z)$. Clearly the sequences of the form $\left(1, z_{i}, z_{i}^{2}, \ldots\right), i=1,2, \ldots, N$, are linearly independent in $l^{\infty}$ and each is in $\left[S^{n} x_{0}\right]^{\perp}$. It follows that $\left[S^{n} x_{0}\right]^{\perp}$ contains

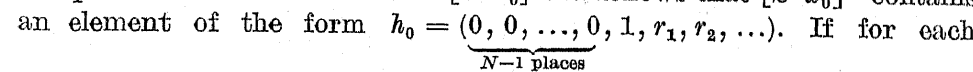
$n=0,1,2, \ldots$ we set $f_{n}=\frac{1}{b_{N}} S^{n+1} h_{0}$, then clearly $\sup _{n}\left\|f_{n}\right\|<+\infty$ and $\left\langle f_{n}, S^{m} x_{0}\right\rangle=\delta_{n m}$ for all $m, n$. That is, $\left\{S^{n} x_{0}, f_{n}\right\}_{n=0}^{\infty}$ is a bounded biorthogonal system and henoe, by Theorem 2.1, a basic sequence in $l^{2}$.

Thus the multiplicity of the zeros of $p(z)$ which lie on the circle $|z|=1$. determine whether or not $\left\{S^{n} x_{0}\right\}$ is basic in $l^{1}$. Our next result shows that these zeros also uniquely determine the similaxity properties of the basic sequence $\left\{S^{n} x_{0}\right\}$.

Theorem 3.5. Let $x_{0}=\left(1, a_{1}, \ldots, a_{N}, 0,0,0, \ldots\right), y_{0}=\left(1, b_{1}, b_{2}, \ldots\right.$ $\left.\ldots, b_{M I}, 0,0, \ldots\right)$, and suppose $\left\{S^{n} x_{0}\right\}$ and $\left\{S^{n} y_{0}\right\}$ are basic sequences in $l^{1}$. Then $\left\{S^{n} x_{0}\right\}$ and $\left\{S^{n} y_{0}\right\}$ are similar if and only if the polynomials

$$
p(z)=1+a_{1} z+\ldots+a_{N} z^{N} \text { and } q(z)=1+b_{1} z+\ldots+b_{M} z^{M}
$$

have exactly the same aeros on the oirole $|z|=1$.

Proof. As in Theorem 3.4. we may assume that $p(z)$ and $q(z)$ have only simple zeros, all of which lio on the circle $|z|=1$. Thus we need only to show that if $\left\{S^{n} x_{0}\right\}$ and $\left\{S^{n} y_{0}\right\}$ are similar, then $p(z)=q(z)$.

To do this, recall that $\left\{S^{n} x_{0}\right\}$ is similar to $\left\{S^{n} y_{0}\right\}$ if and only if there are constants $K_{1}$ and $K_{2}$ for which

$$
\left\|\sum_{n=0}^{r} d_{n} S^{n} x_{0}\right\|_{1} \leqslant K_{1}\left\|\sum_{n=0}^{r} d_{n} S^{n} y_{0}\right\|_{1} \leqslant K_{2}\left\|\sum_{n=0}^{r} a_{n} S^{n} x_{0}\right\|_{1}
$$

for all $r$ and all $\left\{d_{n}\right\}_{n=0}^{r}$.

Now suppose $z_{0}$ is a root of $q(z)$ which is not a root of $p(z)$. Then for $r>2 M r$ ono oasily checks that

$$
\begin{gathered}
\left\|\sum_{n=10}^{r}\left(1 / z_{0}^{n}\right) S^{m} y_{0}\right\|_{1}=\|\left(1, b_{1}+1 / z_{0}, \ldots, b_{M}+b_{M-1} / z_{0}+\ldots+1 / z_{0}^{M},\right. \\
b_{M} / z_{0}+\ldots+1 / z_{0}^{M+1}, \ldots, b_{M} / z_{0}^{r-M}+\ldots+1 / z_{0}^{r}, \\
b_{M} / z_{0}^{r-M+1}+\ldots+b_{1} / z_{0}^{r}, b_{M} / z_{0}^{r-M+2}+\ldots+b_{2} / z_{0}^{r}, \ldots \\
\left.\ldots, b_{M} / z_{0}^{r}, 0,0, \ldots\right) \|_{1} .
\end{gathered}
$$

Sinco $z_{0}$ is a root of $q(z)$, this is oqual to

$$
\begin{aligned}
\|\left(1, b_{1}+1 / \tilde{z}_{0}, \ldots, b_{M-1}+b_{M-2} / z_{0}+\ldots+1 / z_{0}^{M-1}, 0,0, \ldots, 0\right. \\
\\
\left.b_{M} / z_{0}^{r-M M+1}+\ldots+b_{1} / z_{0}^{r}, \ldots, b_{M} / z_{0}^{r}, 0,0, \ldots\right) \|_{1} \leqslant 2 A M
\end{aligned}
$$

where $A=\max \left|x+b_{1} z_{0}+\ldots+b_{n} z_{0}^{n}\right|$, since $\left|z_{0}\right|=1$.

$$
\text { oasuners }
$$

On the other hand, a similar analysis shows that for $r>2 M$ we have $\left\|\sum_{n=0}^{r} 1 / i_{0}^{n} S^{n} x_{0}\right\|_{1} \geqslant(r-2 M) \cdot\left|p\left(z_{0}\right)\right|$. Thus $\left\|\sum_{n=0}^{r} 1 / z_{0}^{n} S^{n} x_{0}\right\|_{1} \rightarrow+\infty$ as $r \rightarrow+\infty$ whilo $\left\|\sum_{n=01}^{r} 1 / \approx_{0}^{n} S^{n} x_{0}\right\|_{1}$ is bounded, a contradiction to our earlier inequalities. Thus if: $\left\{S^{n \prime \prime} x_{0}\right\}$ and $\left\{S^{n} y_{0}\right\}$ are similar, overy root of $q(z)$ is a root of $p(z)$, and vice versal (recull our assumptions on $p(z)$ and $q(z) !)$ and the theorem is provod.

Thoorom 3.5 shows that there aro infinitely many non-similar basic sequences in $l^{1}$ of the form $\left\{S^{n} x_{0}\right\}$ (in contrast to the situation in any other space $X$ ). Trowever, the meriety of these basic sequences is still ribther limited as the following rosult shows.

Recall that ab basic sequence $\left\{x_{n}\right\}$ in a $B$ anach space $X$ for which

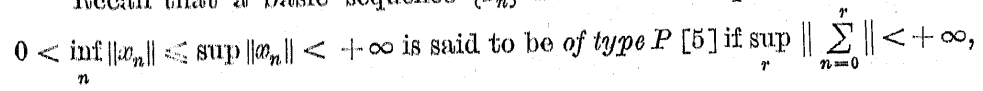


and of type $P^{*}[5]$ if there exists an element $t \in X^{*}$ for which $\left\langle f, x_{n}\right\rangle=1$ for all $n$. It turns out that if $x_{0}$ is finitely non-zero and $\left\{S^{n} x_{0}\right\}$ is a basic sequence in $l^{1}$, then $\left\{S^{n} x_{0}\right\}$ is either of type $P$ or of type $P^{*}$.

THeOREM 3.6. Let $x_{0}=\left(1, a_{1}, \ldots, a_{N}, 0,0, \ldots\right), p(z)=1+a_{1} z+\ldots$ $\ldots+a_{N} z^{N}$, and suppose $\left\{S^{n} x_{0}\right\}$ is a basic sequence in $l^{1}$. Then $\left\{S^{n} x_{0}\right\}$ is

(i) of type $P$ if and only if $p(1)=0$,

(ii) of type $P^{*}$ if and only if $p(\mathbf{1}) \neq 0$.

Proof. (i): For any $r \geqslant N$

$$
\begin{aligned}
&\left\|\sum_{n=0}^{r} S^{n} x_{0}\right\|_{1}=\|\left(1,1+a_{1}, \ldots, \sum_{n=0}^{N-1} a_{n},\right. \underbrace{\sum_{n=0}^{N} a_{n}, \sum_{n=0}^{N} a_{n}, \ldots, \sum_{n=0}^{N} a_{n}}_{n=0}, \\
&\left.\sum_{n=1}^{N} a_{n}, \sum_{n=2}^{N} a_{n}, \ldots, a_{N}, 0,0, \ldots\right) \|_{1} .
\end{aligned}
$$

Therefore we see that $\left\{S^{n} x_{0}\right\}$ is of type $P$. in $l^{1}$ if and only if $\sum_{n=0}^{N} a_{n}=0$, i.e. if and only if $p(1)=0$.

(ii): If $p(1) \neq 0$, then the sequence $F=(1 / p(1), 1 / p(1), \ldots) \in l^{\infty}$ and it is obvious that $\left\langle F, S^{n} x_{0}\right\rangle=1$ for all $n$. That is, $\left\{S^{n} x_{0}\right\}$ is of type $P^{*}$ in $l^{1}$.

On the other hand, if $\left\{S^{n} x_{0}\right\}$ is of type $P^{*}$, then clearly it is not of type $P$ and so by (i) it must be that $p(1) \neq 0$.

By using essentially the same techniques as we have been using throughout this section, one can also prove a number of results which similarly characterize the possible types of basic sequences $\left\{S^{n} x_{0}\right\}$ which may arise. Among these we mention here (without proof) only the following.

Recall that a basic sequence $\left\{x_{n}\right\}$ in a Banach space $X$ for which $0<\inf _{n}\left\|x_{n}\right\| \leqslant \sup _{n}\left\|x_{n}\right\|<+\infty$ is said to be boundedly complete [5] if whenever $\sup _{r}\left\|\sum_{n=0}^{r} a_{n} x_{n}\right\|<+\infty$, then $\sum_{n=0}^{\infty} a_{n} x_{n}$ converges in $X$.

THEOREM 3.7. If $\left\{S^{n} x_{0}\right\}$ is a basio sequence in $l^{1}$, then $\left\{S^{n} x_{0}\right\}$ is boundedly complete if and only if it is similar to the unit vector basis $\left\{e_{n}\right\}$ for $l^{1}$.

Throughout this paper we have been studying conditions under which $\left\{S^{n} x_{0}\right\}$ is a basic sequence in $X$ (i.e. a basis for $\left[S^{n} x_{0}\right]$ in $X$ ) as opposed to the more restrictive problem of when $\left\{S^{n} x_{0}\right\}$ is actually a basis for all of $X$. It is now a simple matter to answer this latter question.

THEOREM 3.8. If $x_{0}=\left(1, a_{1}, a_{2}, \ldots, a_{N}, 0,0, \ldots\right)$, then the following are equivalent:

(i) $\left\{S^{n} x_{0}\right\}$ is a basis for $X$.

(ii) $\left\{S^{n} x_{0}\right\}$ is a basis for $X$ which is similar to the basis $\left\{e_{n}\right\}$ for $X$. (iii) The polynomial $p(z)=1+a_{1} z+\ldots+a_{N} z^{N}$ has no zero in the unit dise $|z| \leqslant 1$.

Proof. The spectrum of the shift operator $S$ on $X$ is the unit disc. Therefore the spectral mapping theorem applied to the operator $T=I+$ $+a_{1} S+a_{2} S^{2}+\ldots+a_{N} S^{N}$ shows that if $p(z)$ has no zero in the disc $|z| \leqslant 1$, then $T$ is invertible, and since $S^{n} x_{0}=T e_{n}$, we see that (iii) $\Rightarrow$ (ii). Obviously (ii) $\Rightarrow$ (i), so we need only to prove that (i) $\Rightarrow$ (iii).

To do this, suppose $\left\{S^{n} x_{0}\right\}$ is a basis for $X$. If $p(z)$ has a zero $z_{0}$ with $\left|z_{0}\right|<1$, then the sequence $\left(1, z_{0}, z_{0}^{2}, \ldots\right)$ is in $\left[S^{n} x_{0}\right]^{\perp} \subset X^{*}$ (for any $X$ ), a contradiction to the fact that $\left[S^{n} x_{0}\right]=X$. Therefore we see that $p(z)$ can have no zero in the open disc $|z|<1$.

If $X \neq l^{1}$, by Theorem $3.2 p(z)$ also has no zero on the circle $|z|=1$ and we are done. If $X=l^{1}$ and $z_{0}$ is a zero of $p(z)$ with $\left|z_{0}\right|=1$, then again the sequence $\left(1, z_{0}, z_{0}^{2}, \ldots\right)$ is in $\left[S^{n} x_{0}\right]^{\perp} \subset l^{\infty}$ and we have reached the same contradiction. In any case, then, the theorem is proved.

Let us conclude this section by describing the subspaces $\left[S^{n} x_{0}\right]_{n=0}^{\infty}$ of $X$ which are spanned by sequences of the form $\left\{S^{n} x_{0}\right\}_{n=0}^{\infty}$ (whether or not this last is a bassic sequence).

To do this, several observations are helpful. If $x_{0}=\left(1, a_{1}, a_{2}, \ldots\right.$ $\ldots, a_{N}, 0,0, \ldots$ ), then (as we have noted many times before) $S^{n} x_{0}=T e_{n}$ for all $n$, where $T=I+a_{1} S+\ldots+a_{N} S^{N}$. Hence $\left[S^{n} x_{0}\right]^{\perp}=K$, where $K$ denotes the kernel of $T^{*} \subset X^{*}$. If we factor $T$ as $T=\left(I-b_{1} S\right)\left(I-b_{2} S\right) \ldots$ $\ldots\left(I-b_{N} S\right)$, where $\left\{1 / b_{i}\right\}_{i=1}^{N}$ is the set of zeros of $p(z)$ counted according to their multiplicity, then $K=\operatorname{ker}\left(I-b_{1} S^{*}\right) \ldots\left(I-b_{N} S^{*}\right)$ and hence $\operatorname{dim} K \leqslant N$

Since $I-b_{i} s^{*}$ is invertible for $\left|b_{i}\right|<1$ (for all $X$ ), we see that $K$ is actually the kernel of the operator $\prod_{i \in \sigma}\left(I-b_{i} S^{*}\right)$, where $\sigma=\left\{i|| b_{i} \mid \geqslant 1\right\}$. Also note that several of the numbers $b_{i}$ may be identical.

In order to treat the case $X=l^{1}$, we need the following result.

LEMCMA 3.9. If $|a|=1$ and $\left(I-a S^{*}\right)^{2} h_{0}=0$ for some $h_{0} \in l^{\infty}$, then $\left(I-a S^{*}\right) h_{0}=0$.

Proof. It is easy to see that $\operatorname{ker}\left(I-a S^{*}\right)=\left[\left(1,1 / a, 1 / a^{2}, \ldots\right)\right] \subset l^{\infty}$. Thorefore if $\left(I-a S^{*}\right)^{2} h_{0}=0$, then $\left(I-a S^{*}\right) h_{0}$ must be of the form $\left(\lambda, \lambda / a, \lambda / a^{2}, \ldots\right)$ for some number $\lambda$. That is, if $h_{0}=\left(y_{0}, y_{1}, y_{3}, \ldots\right)$, then $y_{0}-a y_{1}=\lambda, y_{1}-a y_{2}=\lambda / a, \ldots, y_{n}-a y_{n+1}=\lambda / a^{n}$. Successively solving these equations, we see that, in general, $y_{n}=y_{0} / a^{n}-n \lambda / a^{n}$ for $n=1,2, \ldots$ Since $h_{0}=\left\{y_{n}\right\}$ is in $l^{\infty}$, it must be that $\lambda=0$. That is, $\left(I-a s^{*}\right) h_{0}=0$ and the lemma is proved.

It follows from Lemma 3.9 that when computing $K$ in the case $X=l^{1}$, we may ignore any repeated factors of the form $I-b_{i} S^{*}$, where $\left|b_{i}\right|=1$. In the case where $X \neq l^{1}$, we may ignore any such factor since each gives rise to a one-to-one operator $I-b_{i} S$ (Lemma 2.3). Thus if $n_{1}$ is the number 
of zeros of $p(z)$ in the open disc $|z|<1$ (counting multiplicities) and $n_{2}$ is the number of distinct zeros of $p(z)$ on the circle $|z|=1$, then it is clear that if $X \neq l^{1}, \operatorname{dim} K=\operatorname{dim}\left[S^{n} x_{0}\right]^{\perp} \leqslant n_{1}$, while if $X=l^{1}, \operatorname{dim} K$ $=\operatorname{dim}\left[S^{n} x_{0}\right]^{\perp} \leqslant n_{1}+n_{2}$.

The next theorem shows that equality actually holds in both of these cases. More importantly, the proof gives a construction of a basis for $\left[S^{n} x_{0}\right]^{\perp}$. As usual, let $f^{\left(k_{k}\right)}$ denote the $k$ th derivative of $f$ on the unit dise.

THEOREM 3.10. If $X \neq l^{1}$, then $\operatorname{dim}\left[S^{n} x_{0}\right]^{\perp}=n_{1}$, while if $X=l^{1}$, $\operatorname{dim}\left[S^{n} x_{0}\right]^{\perp}=n_{1}+n_{2}$.

Proof. Let $g(z)=1+z+z^{2}+\ldots$ and let $\left\{b_{i}^{(K)}\right\}_{i=1}^{\infty}$ be the sequence of coefficients in the power series expansion of $g^{(K-1)}(z)$ for $K=1,2,3, \ldots$ If $z_{0}$ is a zero of $p(z)$ of multiplicity $m$ and lying in the open dise $|z|<1$, then since $\left[z^{r} p\right]^{(k)}\left(z_{0}\right)=0$ for $0 \leqslant 7 \leqslant m-1$ and all $r=0,1,2, \ldots$, it is clear that the $m$ sequences

$$
\begin{aligned}
& h_{1}=\left(b_{0}^{(1)}, b_{1}^{(1)} z_{0}, b_{2}^{(1)} z_{0}^{2}, \ldots\right) \\
& h_{2}=\left(0, b_{0}^{(2)}, b_{1}^{(2)} z_{0}, \ldots\right) \\
& h_{3}=\left(0,0, b_{0}^{(3)}, b_{1}^{(3)} z_{0}, \ldots\right) \\
& \vdots \\
& h=(\underbrace{\left.0,0, \ldots, 0, b_{0}^{(m)}, b_{1}^{(m)} z_{0}, b_{2}^{(m)} z_{0}^{2}, \ldots\right)}_{m-1 \text { places }} .
\end{aligned}
$$

form a linearly independent set in $\left[S^{n} x_{0}\right]^{\perp}$. This is so for every zero of $p(z)$ in $|z|<1$, and since the $n_{1}$ sequences thus generated aro linearly independent in $X^{*}$, we see that for any $X \operatorname{dim}\left[S^{n} x_{0}\right]^{\perp} \geqslant n_{x}$. Therefore in the case $X \neq l^{1}$ we must actually have $\operatorname{dim}\left[S^{n} x_{0}\right]^{\perp}=n_{1}$ according to our earlier inequality.

In the case $X=l^{1}$, if $z_{0}$ is a zero of $p(z)$ on the circle $|z|=1$, then the sequence $\left(1, z_{0}, z_{0}^{2}, \ldots\right)$ is in $\left[S^{n} x_{0}\right]^{\perp} \subset l^{\infty}$. In this way the $n_{2}$ distinct zeros of $p(z)$ on the circle $|z|=1$ generate another set of $n_{2}$ linearly independent elements in $\left[S^{n} x_{0}\right]^{\perp}$ and it follows that $\operatorname{dim}\left[S^{n} x_{0}\right]^{\perp} \geqslant n_{1}+n_{2}$. Together with our earlier inequality this says that $\operatorname{dim}\left[S^{n} x_{0}\right]^{\perp}=n_{1}+n_{2}$ and the proof is complete.

Now using the construction of a basis for $\left[S^{n} x_{0}\right]^{\perp}$ given in the preceding proof of Theorem 3.10, we obtain the following characterization of [ $S^{n} x_{0}$ ] in $X$.

THEOREM 3.11. Let $x_{0}=\left(1, a_{1}, \ldots, a_{N}, 0,0, \ldots\right)$ and $p(z)=1+$ $+a_{1} z+\ldots+a_{N} z^{N}$. Then

(i) If $X \neq l^{1}$, the subspace $\left[S^{n} x_{0}\right]$ of $X$ is equal to the set of all sequenses $\left(b_{0}, b_{1}, b_{2}, \ldots\right)$ in $X$ for which the function $f(z)=b_{0}+b_{1} z+b_{2} z^{2}+\ldots$ has the property that $f^{(\mathbb{E})}(z)=0$ if $p^{(\bar{K})}(z)=0$ for $|z|<1$.

(ii) If $X=l^{1}$, the subspace $\left[S^{n} x_{0}\right]$ of $l^{1}$ is the set of all sequences $\left(b_{0}, b_{1}, \ldots\right)$ in $l^{1}$ for which the funotion $f(z)=b_{0}+b_{1} z+\ldots$ has the property that $f^{(K)}(z)$ $=0$ if $p(z)=0$ for $|z|<1$ and $f(z)=0$ if $p(z)=0$ for $|z|=1$.
Remark. The characterization given in Theorem 3.11 is valid regardless of whether or not $\left\{S^{n} x_{0}\right\}$ is basic in $X$. Moreover, this characterization admits an obvious generalization to the case in which $x_{0}$ is arbitrary in $X$ (though the proof is considerably more difficult). As such it is very useful in obtaining information concerning the basic sequence properties of $\left\{S^{n} x_{0}\right\}$ in this general setting. Details will be given in a forthcoming paper.

\$4. Generalizations. If one considers the problems treated here in the genersil setting in which $x_{0}$ is allowed to be an arbitiary member of $X$, then the difficulties oncountered in attempting to answer the same questions become immediately obvious and in most cases quite formidalole (see [2], e.g.). If we restrict ourselves to the case $X=l^{1}$, there is available enough structure to be able to make some progress on these problems although many inviting questions remain. We mention here several of the more elementary results in this area. Others will be given in a subsequent paper.

The question of when $\left\{S^{n} x_{0}\right\}$ is a basis for $l^{1}$ has a surprisingly simple answer. Under the weakest of all possible conditions, namely that $\left\{S^{n} x_{0}\right\}$ spans $l^{1}$, the sequence $\left\{S^{n} x_{0}\right\}$ is a basis.

THeornm 4.1. Let $x_{0}=\left(1, a_{1}, a_{2}, \ldots\right)$ be in $l^{1}$. Then the following are equivatent:

(i) $\left\{S^{n} x_{0}\right\}$ is a basis for $l^{1}$ similar to $\left\{e_{n}\right\}$.

(ii) $\left\{S^{n} x_{0}\right\}$ is a basis for $l^{1}$.

(iii) $\left[S^{n} x_{0}\right]_{n=0}^{\infty}=l^{1}$.

Proof. Olearly (i) $\Rightarrow$ (ii) $\Rightarrow$ (iii). We prove that (iii) $\Rightarrow$ (i).

As we have noted several times previously, if $f(z)=1+a_{1} z+a_{2} z^{2}+\ldots$ and $z_{0}$ is a zero of $f(z)$ in the disc $|z| \leqslant 1$, then the sequence $\left(1, z_{0}, z_{0}^{2}, \ldots\right)$ is a member of $\left[S^{n} x_{0}\right]^{\perp}$. Hence (iii) implies that $f(z) \neq 0$ for all $z$ in the dise $|z| \leqslant 1$ and by the Wiener-Lery theorem $[3]$, p. $97 f^{-1}(z)=1+$ $+c_{1} z+o_{2} z^{2}+\ldots$ with $\left\{c_{i}\right\}_{i=0}^{\infty}$ in $l^{1}$.

According to our earlier remarks, the sequence $\left\{f_{n}\right\}_{n=0}^{\infty} \subset l^{\infty}$ defined by $f_{n}=\left(c_{n}, c_{n-1}, \ldots, c_{1}, 1,0,0, \ldots\right)$ is biorthogonal to $\left\{S^{n} x_{0}\right\}$ and clearly $\left\{f_{n}\right\}$ has the property that if $\left\langle f_{n}, \infty\right\rangle=0$ for all $n=0,1,2, \ldots$, then $x=0$.

Now suppose $x$ is in $l^{2}$. Then the sequenco $\left\{\left\langle f_{n}, \infty\right\rangle\right\}_{n=0}^{\infty}$ is also in $l^{1}$ since it is simply the Oauchy product of the sequences $\left\{c_{n}\right\}_{n=0}^{\infty}$ and $\left\{\left\langle x, e_{n}\right\rangle\right\}_{n=010}^{\infty}$, both of which are in $l^{1}$. Therefore the series $\sum_{n=0}^{\infty}\left\langle f_{n}, \infty\right\rangle S^{n} x_{0}$ is absolutely convergent in $l^{1}$, say to some $y$. Since $\left\langle f_{n}, y\right\rangle=\left\langle f_{n}, x\right\rangle$ for all $n$, it follows that $y=x$ and therefore that $\sum_{n=0}^{\infty}\left\langle f_{n}, x\right\rangle S^{n} x_{0}=x$ for all $x$ 
in $l^{1}$. That is, $\left\{S^{n} x_{0}\right\}$ is a basis for $l^{1}$ which, as our proof shows, is similar to $\left\{e_{n}\right\}$.

Using essentially the same icleas we can prove:

TheOREMr 4.2. If $x_{0}=\left(1, a_{1}, a_{2}, \ldots\right) \in l^{2}$ and $f(z)=1+a_{1} z+a_{2} z^{2}+\ldots$ with $f(z) \neq 0$ for all $z$ in the unit diso $|z| \leqslant 1$, then $\left\{S^{n} x_{0}\right\}$ is a basis for $l^{1}$ which is similar to $\left\{e_{n}\right\}$.

Proof. As in the proof of Theorem 4.2, our assumptions imply that $f^{-1}(z)=1+c_{1} z+\ldots$ for $\left\{c_{i}\right\}_{i=0}^{\infty}$ in $l^{1}$. Therefore the operator $Q=I+c_{1} S+$ $+c_{2} S^{2}+\ldots$ is a bounded linear operator on $l^{1}$ and clearly $Q T=T Q=I$. That is, $T$ is invertible on $l^{2}$ and since $T e_{n}=S^{n} x_{0}$, the theorem is proved.

Corollary 4.3. I $x_{0} \in l^{1}$, the following are equivalent:

(i) $\left\{S^{n} x_{0}\right\}$ is a basis for $l^{1}$ similar to $\left\{\boldsymbol{e}_{n}\right\}$.

(ii) $\left\{S^{n} x_{0}\right\}$ is a basis for $\eta^{1}$.

(iii) The function $f(z)=1+a_{1} z+a_{2} z^{2}+\ldots$ has no zero on the diso $|z| \leqslant 1$.

Finally we state without proof a more general version of Theorem 3.1 (for the case $X=l^{1}$ ) which settles the question of similaxity of $\left\{S^{n} x_{0}\right\}$ and $\left\{e_{n}\right\}$ in $l^{1}$.

THEOREM 4.4. Let $x_{0}=\left(1, a_{1}, a_{2}, \ldots\right) \in l^{1}$ and $f(z)=1+a_{1} z+a_{2} z^{2}+\ldots$ Then $\left\{\mathcal{S}^{n} x_{0}\right\}$ is a basic sequenoe in $l^{1}$ whioh is similar to $\left\{e_{n}\right\}$ if and only if $f(z) \neq 0$ for all $\approx$ on the unit circle $|z|=1$.

The proof uses essentially the same ideas outlined above along with certain estimates on the norms of linear combinations of $\left\{\mathcal{S}^{n} x_{0}\right\}$ and will be given in a subsequent paper devoted to more general problems in this area.

\section{References}

[1] I. C. Gohberg and M. G. Krein, Introduction to the theory of linear nonselfadjoint operators, Trans. Math. Monographs, Vol. 18, Amer. Math. Soc., Providence, R. I. 1969 .

[2] H. Hels on and G. Szeg ö, A problem in prediction theory, Ann. Mat. Pura Appl. 51 (1960), 107-138.

[3] K. Hoffman, Banach spaces of analytio functions, Prontico Hall, Englewood Cliffs, N. J. 1962.

[4] J. Lindenstrauss and L. Tzafriri, Olassical Banaoh spaces, Springer-Verlag, Berlin 1973.

[5] I. Singer, Bases in Banach spaces I, Springer-Verlag, Berlin 1970.

[6] A. Szankowski and M. Zippin, A stability property of the unit veotor basis of $l_{p}$, Israel J. Math., 20 (1975), 216-227. VIRGINIA POLYTECHNIO INSTITUTE AND STATE UNTVERSTTY
BLACKSBURG, VIRGINIA 24061

Received September 6, 1978

(1465)
1. refiroment of the Mllson-Szegö theorem and the detcrmination of the extremal measures

by

RODRIGO AROCENA (Caracas)

Alostraet. Iset $R_{y}$ bo the set of measures on the unit eirche which satisfy the M. Nins inoquality for the Hilbort transform with constant $M . R_{M}$ is determined by an askeciated clats $I_{M}$ of analytic functions. Wo give a geometric characterization of the elements of: $T_{M}$ ind derive a refinement; of the Helson-Szegö theorem. The extrmal mentures in the cono $T_{M}$ are determined. Our basic result is the constraction of a subset of extremal measures by moans of which every elomont in $R_{M I}$ can be naturally obtatined.

1. Introduction. Tut $T$ denoto the wnit circle, $\hat{f}$ the Fourier transform of $f \in L_{1}(T)$ and $\tilde{f}$ its conjugate function. If $M>1$ is a fixed constant and $\mu \geqslant 0$ a messure on $T$, the Filbert transform, we shall write $\mu \in R_{M}$ if:

$$
\int_{T^{\prime}}|f|^{2} d \mu \leqslant M \int_{T^{T}}|f|^{2} d \mu, \quad \forall f \in L^{2}(\mu) \cap t^{2} .
$$

Set $R=\bigcup_{M>1} R_{M}$. Trelson and Szegö proved [3] that $\mu \in R$ iff $\mu$ is absolutely continuous with respect to Lebesgue measure, $d \mu=w(x) d x$, $\operatorname{sind}$

(I.2) $\quad w=\exp (u+\tilde{v}), \quad u, v \in L^{\infty}, \quad\|v\|_{\infty}<\pi / 2$.

Consequently, from now on we shall write $w \in R_{M}$ iff $\mu \in R_{M}$. Cotlar and Sadosky proved [2] that $w \in R_{M}$ iff there exists $h \in H^{1}(T)$ such that:

$$
-4 M w^{2}-2(M+1) \operatorname{Re}(h) w-|h|^{2} \geqslant 0 \text {, a.e. }
$$

In this priper we study first those functions $h$ that, by (I.3), characterize $h_{M}$. Then we statio a version of Melson-Szegö theorem for each $R_{M}$, and, in particular, a simple proof of (T.2), deduced from (T.3). Finally wo dotermine the extremal rays of the cone $R_{M}$. Our basic result is:

Tumoxwa 1. Let $g=g_{1}+i g_{2} \neq 0$ be a function of $H^{1}(T)$ with $\hat{g}(0)=0$ and $\tau(g)=\left(v_{0}, u_{0}\right)$, where

$$
\begin{aligned}
& v_{0}=\operatorname{artig}\left\{(M I-1) g_{1} /\left[(M C+1)^{2} g_{2}^{2}+4 M g_{1}^{2}\right]^{1 / 2}\right\}, \\
& u_{0}=\operatorname{arch}\left(\frac{M T+1}{2 \sqrt{M}} \cos v_{0}\right) \cdot\left(\chi_{\left\{g_{2}>0\right\}}-\chi_{\left\{g_{2}<0\right\}}\right) .
\end{aligned}
$$

